Freiburg-THEP 06/11

DFTT-13/2006

\title{
Magnetic dipole operator contributions to the photon energy spectrum in $\bar{B} \rightarrow X_{s} \gamma$ at $O\left(\alpha_{s}^{2}\right)$
}

\author{
H.M. Asatrian ${ }^{a}$, T. Ewerth ${ }^{b}$, A. Ferroglia ${ }^{c}$, \\ P. Gambino ${ }^{d}$, and C. Greub ${ }^{b}$ \\ ${ }^{a}$ Yerevan Physics Institute, 375036 Yerevan, Armenia \\ ${ }^{b}$ Inst. for Theoretical Physics, Univ. Berne, CH-3012 Berne, Switzerland \\ ${ }^{c}$ Physikalisches Institut, Albert-Ludwigs-Universität, D-79104, Freiburg, Germany \\ ${ }^{d}$ INFN Sez. di Torino \& Dip. Fisica Teorica, Univ. di Torino, I-10125, Torino, Italy
}

\begin{abstract}
We compute the $O\left(\alpha_{s}^{2}\right)$ contributions to the photon energy spectrum of the inclusive decay $\bar{B} \rightarrow X_{s} \gamma$ associated with the magnetic penguin operator $O_{7}$. They are an essential part of the ongoing NNLO calculation of this important decay. We use two different methods to evaluate the master integrals, one based on the differential equation approach and the other on sector decomposition, leading to identical results which in turn agree with those of a recent independent calculation by Melnikov and Mitov. We study the numerical relevance of this set of NNLO contributions in the photon energy spectrum and discuss the change of bottom quark mass scheme.
\end{abstract}




\section{Introduction}

More than a decade after their first direct observation, radiative $B$ decays have become a key element in the program of precision tests of the Standard Model (SM) and its extensions. The inclusive decay $\bar{B} \rightarrow X_{s} \gamma$ is particularly well suited to this precision program thanks to the low sensitivity to non-perturbative effects. The present experimental world average [1] for the branching ratio of $\bar{B} \rightarrow X_{s} \gamma$ has a total error of about $6 \%$ and agrees well with the SM prediction, that is subject to a considerably larger uncertainty [2]. In view of the final accuracy expected at the $B$ factories, about $5 \%$, the SM calculation needs to be improved. It presently includes next-to-leading order (NLO) perturbative QCD corrections as well as the leading non-perturbative and electroweak effects (see e.g. [3] for a complete list of references). The calculation of next-to-next-to-leading order (NNLO) QCD effects is currently under way and is expected to bring the theoretical accuracy to the required level.

Among the NNLO QCD contributions, the following have already been computed: i) all relevant $O\left(\alpha_{s}^{2}\right)$ Wilson coefficients [4]; ii) the relevant three loop anomalous dimension matrix $[5,6]$; iii) the $O\left(\alpha_{s}^{2} N_{F}\right)$ corrections to the matrix elements of the operators $O_{1}, O_{2}, O_{7}, O_{8}[7]$; iv) those matrix elements related to the dominant electromagnetic dipole operator $O_{7}$ [8-10]. Among the pieces still missing to date, there are two rather important and difficult ones. The first concerns the four-loop anomalous dimension matrix elements that describe the $O\left(\alpha_{s}^{3}\right)$ mixing of the four-quark operators into $O_{7}$ and $O_{8}$; preliminary results on this have been recently presented [11]. The second concerns the three-loop, $O\left(\alpha_{s}^{2}\right)$, matrix elements of the four-quark operators $\mathrm{O}_{1}$ and $\mathrm{O}_{2}$. These loops contain the charm quark, and the ambiguity associated to the choice of scale for its mass is the main source of uncertainty at NLO [2]. A NNLO calculation of these matrix elements is therefore crucial to reduce the overall theoretical error on the branching ratio [12]. Other missing contributions, like the $O\left(\alpha_{s}^{2}\right)$ matrix elements of $O_{8}$, may also prove essential to reach a high theoretical precision.

In addition to the total branching fraction, the photon energy spectrum in $\bar{B} \rightarrow X_{s} \gamma$ is also a useful observable: it receives both perturbative and non-perturbative contributions and is important for several reasons: i) the precise knowledge of the spectrum is necessary to predict the measured branching ratio since experiments apply a lower cut of $1.8-2.0 \mathrm{GeV}$ on the photon energy in the $B$ center-of-mass frame; ii) it is almost insensitive to new physics; iii) from the moments of the truncated spectrum one can extract relevant information on the parameters of the Heavy Quark Expansion. In particular, a precise value of the $b$ quark mass can be extracted from the mean value $\left\langle E_{\gamma}\right\rangle$ of the photon energy [13, 14]; iv) the measured spectrum gives direct information on the Shape Function that encodes the QCD dynamics in the endpoint region, where non-perturbative contributions dominate and perturbative corrections must be resummed [15]. The NNLO Sudakov resummation was first completed in [16] and there has been recent progress towards NNLO in the multi-scale OPE approach [17]. A detailed knowledge of the Shape Function is useful for the determination of $\left|V_{u b}\right|$ from inclusive semileptonic $B$ decays.

The perturbative contributions to the photon energy spectrum are known at $O\left(\alpha_{s}\right)[18]$ and $O\left(\alpha_{s}^{2} \beta_{0}\right)$ [19] since several years. In this paper we present a calculation of the $O\left(\alpha_{s}^{2}\right)$ photon spectrum induced by the magnetic operator $O_{7}$. This operator gives the dominant contribution to the spectrum at $O\left(\alpha_{s}\right)$. Our results, which were obtained by using two different methods, confirm those of a recent paper [10]. 
Our paper is organized in the following way. In Sec. 2 we present our analytical results for the unnormalized photon energy spectrum and compare with the literature. In this section we also compare numerically the NLO and the NNLO spectra, and study the impact of a change of the bottom quark mass scheme. Sec. 3 is devoted to a detailed description of the techniques used for our calculation. Finally, we give a brief summary in Sec. 4.

\section{Results and applications}

\subsection{Analytical results for the photon energy spectrum}

Within the low-energy effective theory the partonic $b \rightarrow X_{s} \gamma$ decay rate can be written as

$$
\Gamma\left(b \rightarrow X_{s}^{\mathrm{parton}} \gamma\right)_{E_{\gamma}>E_{0}}=\frac{G_{F}^{2} \alpha_{\mathrm{em}} \bar{m}_{b}^{2}(\mu) m_{b}^{3}}{32 \pi^{4}}\left|V_{t b} V_{t s}^{*}\right|^{2} \sum_{i, j} C_{i}^{\mathrm{eff}}(\mu) C_{j}^{\mathrm{eff}}(\mu) G_{i j}\left(E_{0}, \mu\right)
$$

where $m_{b}$ and $\bar{m}_{b}(\mu)$ denote the pole and the running $\overline{\mathrm{MS}}$ mass of the $b$ quark, respectively, $C_{i}^{\text {eff }}(\mu)$ the effective Wilson coefficients at the low energy scale, and $E_{0}$ the energy cut in the photon spectrum. Here we give the result for the function $G_{77}\left(E_{0}, \mu\right)$ corresponding to the self-interference of the electromagnetic dipole operator

$$
O_{7}=\frac{e}{16 \pi^{2}} \bar{m}_{b}(\mu)\left(\bar{s} \sigma^{\mu \nu} P_{R} b\right) F_{\mu \nu}
$$

including $O\left(\alpha_{s}^{2}\right)$ terms as required for NNLO accuracy. Introducing the dimensionless variable

$$
z=\frac{2 E_{\gamma}}{m_{b}}
$$

this function can be written as an integral over the (rescaled) photon energy spectrum $d G_{77}(z, \mu) / d z$, i.e.,

$$
G_{77}\left(E_{0}, \mu\right)=\int_{z_{0}}^{1} \frac{d G_{77}(z, \mu)}{d z} d z
$$

where $z=z_{0}$ corresponds to $E_{\gamma}=E_{0}$. When working to the required precision, the photon energy spectrum can be separated into three different parts,

$$
\frac{d G_{77}(z, \mu)}{d z}=\sum_{n=2}^{4} \frac{d G_{77}^{1 \rightarrow n}(z, \mu)}{d z}
$$

corresponding to the $n$ particles in the final state, namely the $b \rightarrow s \gamma(n=2), b \rightarrow s \gamma g$ $(n=3), b \rightarrow s \gamma g g$ and $b \rightarrow s \gamma q \bar{q}$ (both $n=4, q \in\{u, d, c, s\}$ ) transitions. Furthermore, each individual contribution can itself be written in the form

$$
\begin{aligned}
& \frac{d G_{77}^{1 \rightarrow 2}(z, \mu)}{d z}=f_{2}(\mu) \delta(1-z), \\
& \frac{d G_{77}^{1 \rightarrow 3}(z, \mu)}{d z}=f_{3}(\mu) \delta(1-z)+R_{3}(z, \mu),
\end{aligned}
$$




$$
\frac{d G_{77}^{1 \rightarrow 4}(z, \mu)}{d z}=f_{4}(\mu) \delta(1-z)+R_{4}(z, \mu) .
$$

Consequently, the complete result for the photon energy spectrum is of the form

$$
\frac{d G_{77}(z, \mu)}{d z}=F(\mu) \delta(1-z)+R_{3}(z, \mu)+R_{4}(z, \mu),
$$

with $F(\mu)=f_{2}(\mu)+f_{3}(\mu)+f_{4}(\mu)$. We stress here that the calculation of $d G_{77}(z, \mu) / d z$ boils down to the determination of $R_{3}(z, \mu)$ and $R_{4}(z, \mu)$, because the coefficient $F(\mu)$ in front of the delta-function can then be fixed by the requirement that the total integral

$$
G_{77}(0, \mu)=\int_{0}^{1} \frac{d G_{77}(z, \mu)}{d z} d z
$$

yields the result obtained in [8], which was recently confirmed in [9].

As we discuss the details of the calculation in Sec. 3, we immediately present our final result for the photon energy spectrum (including also the order $\alpha_{s}^{0}$ and $\alpha_{s}^{1}$ pieces):

$$
\frac{d G_{77}(z, \mu)}{d z}=\delta(1-z)+\frac{\alpha_{s}(\mu)}{\pi} C_{F} H^{(1)}(z, \mu)+\left(\frac{\alpha_{s}(\mu)}{\pi}\right)^{2} C_{F} H^{(2)}(z, \mu)+O\left(\alpha_{s}^{3}\right),
$$

where

$$
H^{(2)}(z, \mu)=C_{F} H^{(2, \mathrm{a})}+C_{A} H^{(2, \mathrm{na})}+T_{R} N_{L} H^{(2, \mathrm{NL})}+T_{R} N_{H} H^{(2, \mathrm{NH})} .
$$

The numerical values of the color factors are $C_{F}=4 / 3, C_{A}=3$, and $T_{R}=1 / 2$. Furthermore, $N_{L}$ and $N_{H}$ denote the number of light $\left(m_{q}=0\right)$ and heavy $\left(m_{q}=m_{b}\right)$ quark flavors, that is the total number of quark flavors is $N_{F}=N_{L}+N_{H}$. The functions $H^{(1)}$ and $H^{(2, j)}$ $(j=\mathrm{a}, \mathrm{na}, \mathrm{NL}, \mathrm{NH})$ appearing in (2.9) and (2.10) are given by

$$
\begin{aligned}
H^{(1)}= & -\left(\frac{5}{4}+\frac{\pi^{2}}{3}+L_{\mu}\right) \delta(1-z)-\left[\frac{\ln (1-z)}{1-z}\right]_{+} \\
& -\frac{7}{4}\left[\frac{1}{1-z}\right]_{+}-\frac{z+1}{2} \ln (1-z)+\frac{7+z-2 z^{2}}{4}, \\
H^{(2, \mathrm{a})}= & {\left[8.10798+\left(\frac{29}{8}+\frac{\pi^{2}}{3}\right) L_{\mu}+\frac{1}{2} L_{\mu}^{2}\right] \delta(1-z)+C(z) L_{\mu} } \\
& +\frac{1}{2}\left[\frac{\ln ^{3}(1-z)}{1-z}\right]_{+}+\frac{21}{8}\left[\frac{\ln ^{2}(1-z)}{1-z}\right]_{+} \\
& +\left(\frac{69}{16}+\frac{\pi^{2}}{6}\right)\left[\frac{\ln (1-z)}{1-z}\right]_{+}+\left(\frac{5 \pi^{2}}{12}-\frac{\zeta(3)}{2}+\frac{67}{32}\right)\left[\frac{1}{1-z}\right]_{+} \\
& +\frac{1+2 z-2 z^{2}+z^{3}}{4 z} \ln ^{3}(1-z)+\frac{z^{3}-4 z^{2}+4 z+1}{24(1-z)} B(z) \\
& +\frac{8 z^{6}-46 z^{5}+64 z^{4}-3 z^{3}-27 z^{2}+21 z-9}{24(1-z)^{3} z} A(z)
\end{aligned}
$$




$$
\begin{aligned}
& +\left[\frac{-9+5 z+7 z^{2}+5 z^{3}-3 z^{4}+z^{5}}{24 z}+\frac{\left(z^{2}+8 z-11\right) \ln z}{8(1-z)}\right] \ln ^{2}(1-z) \\
& +\left[\frac{\pi^{2}\left(3 z-1-z^{2}\right)}{12}+\frac{z^{6}-4 z^{5}-8 z^{4}+61 z^{3}-74 z^{2}+13 z+3}{12 z(1-z)} \ln (2-z)\right. \\
& \left.+\frac{32 z^{4}-156 z^{3}+98 z^{2}+95 z+35}{48}\right] \ln (1-z)+\frac{11-2 z-9 z^{2}+2 z^{3}}{4(z-1)} \times \\
& \times \operatorname{Li}_{2}(1-z) \ln (1-z)+\frac{-32 z^{5}+144 z^{4}+68 z^{3}+z^{2}-297 z-36}{96 z} \\
& -\frac{\pi^{2}\left(z^{5}-3 z^{4}-21 z^{3}+41 z^{2}+19 z-6\right)}{72 z}+\frac{z^{3}+3 z^{2}+10 z-16}{4(1-z)} \zeta(3) \\
& +\left[\frac{z^{6}-4 z^{5}-8 z^{4}+61 z^{3}-74 z^{2}+13 z+3}{12 z(1-z)}+\frac{-3+2 z-2 z^{2}+z^{3}}{2(z-1)} \ln (1-z)\right] \times \\
& \times \mathrm{Li}_{2}(z-1)-\frac{11+4 z-17 z^{2}+4 z^{3}}{4(z-1)} \mathrm{Li}_{3}(1-z)-2(1-z)^{2} \operatorname{Li}_{3}(z-1) \\
& +\frac{11-8 z-z^{2}}{4(1-z)} \operatorname{Li}_{3}(z) \\
& H^{(2, \mathrm{na})}=-\left[13.7256+\left(\frac{211}{36}+\frac{11 \pi^{2}}{18}\right) L_{\mu}+\frac{11}{12} L_{\mu}^{2}\right] \delta(1-z)-\frac{11}{6} C(z) L_{\mu} \\
& +\frac{11}{8}\left[\frac{\ln ^{2}(1-z)}{1-z}\right]_{+}+\left(\frac{95}{144}+\frac{\pi^{2}}{12}\right)\left[\frac{\ln (1-z)}{1-z}\right]_{+} \\
& +\left(\frac{\zeta(3)}{4}-\frac{905}{288}+\frac{17 \pi^{2}}{72}\right)\left[\frac{1}{1-z}\right]_{+}+\frac{z(z-2)^{2}+1}{48(z-1)} B(z) \\
& +\frac{(z+1)\left(15-57 z+73 z^{2}-29 z^{3}+2 z^{4}\right)}{48(z-1)^{3}} A(z) \\
& +\frac{z^{6}-4 z^{5}-2 z^{4}+54 z^{3}-74 z^{2}+z-6}{24(z-1) z}\left[\ln (1-z) \ln (2-z)+\operatorname{Li}_{2}(z-1)\right] \\
& -\frac{(z-1)^{2}}{8} \ln ^{3}(1-z)-\frac{(z+2)}{48}\left(z^{3}-5 z^{2}+9 z-35\right) \ln ^{2}(1-z) \\
& +\left[\frac{\pi^{2}\left(z^{2}-z+3\right)}{24}-\frac{12 z^{5}-156 z^{4}+57 z^{3}+545 z^{2}+74 z+72}{144 z}\right] \ln (1-z) \\
& -\left[\frac{(z-3) z}{4} \operatorname{Li}_{2}(1-z)+\frac{z^{3}-2 z^{2}+2 z-3}{4(z-1)} \operatorname{Li}_{2}(z-1)\right] \ln (1-z) \\
& +\frac{12 z^{4}-138 z^{3}-628 z^{2}+659 z+671}{288}+\frac{\pi^{2}\left(z^{5}-3 z^{4}-3 z^{3}+34 z^{2}-24 z+3\right)}{144 z} \\
& +\frac{(z-3) z}{2} \operatorname{Li}_{3}(1-z)+(z-1)^{2} \operatorname{Li}_{3}(z-1)+\frac{z^{2}+3 z+5}{8} \zeta(3)
\end{aligned}
$$

with

$$
A(z)=2 \ln (1-z)\left((z-1)^{2}+\ln [z(2-z)]\right)+\operatorname{Li}_{2}\left((z-1)^{2}\right)-(z-1)^{2},
$$




$$
\begin{aligned}
B(z)= & 24 \operatorname{Li}_{3}\left(\frac{1}{2-z}\right)+2 \ln (2-z)\left(6 \ln ^{2}(1-z)-2 \ln ^{2}(2-z)+\pi^{2}\right) \\
& +12 \operatorname{Li}_{3}(z)-12 \operatorname{Li}_{3}\left(\frac{z}{2-z}\right)+12 \operatorname{Li}_{3}\left(\frac{z}{z-2}\right)-15 \zeta(3), \\
C(z)= & \frac{7}{4}\left[\frac{1}{1-z}\right]_{+}+\left[\frac{\ln (1-z)}{1-z}\right]_{+}+\frac{1}{2}(1+z) \ln (1-z)+\frac{1}{4}\left(2 z^{2}-z-7\right) .
\end{aligned}
$$

Finally,

$$
\begin{aligned}
H^{(2, \mathrm{NL})=} & {\left[\frac{631}{432}+\frac{91 \pi^{2}}{216}+\frac{\zeta(3)}{3}+\left(\frac{14}{9}+\frac{2 \pi^{2}}{9}\right) L_{\mu}+\frac{1}{3} L_{\mu}^{2}\right] \delta(1-z)+\frac{2}{3} C(z) L_{\mu} } \\
& -\frac{1}{2}\left[\frac{\ln ^{2}(1-z)}{1-z}\right]_{+}-\frac{13}{36}\left[\frac{\ln (1-z)}{1-z}\right]_{+}+\left(\frac{85}{72}-\frac{\pi^{2}}{18}\right)\left[\frac{1}{1-z}\right]_{+} \\
& +\frac{z^{2}-3}{6(1-z)}\left(\mathrm{Li}_{2}(z)-\frac{\pi^{2}}{6}\right)-\frac{1+z}{4} \ln ^{2}(1-z)-(1+z) \frac{\pi^{2}}{36} \\
& -\frac{6 z^{3}-25 z^{2}-z-18}{36 z} \ln (1-z)+\frac{38 z^{2}-55 z-49}{72}, \\
H^{(2, \mathrm{NH})}= & {\left[\frac{3563}{648}-\frac{29 \pi^{2}}{54}-\frac{1}{3} \zeta(3)+\left(\frac{14}{9}+\frac{2 \pi^{2}}{9}\right) L_{\mu}+\frac{1}{3} L_{\mu}^{2}\right] \delta(1-z)+\frac{2}{3} C(z) L_{\mu} . }
\end{aligned}
$$

Here, $L_{\mu}=\ln \left(\mu / m_{b}\right), \operatorname{Li}_{3}(z)=\int_{0}^{z} \mathrm{~d} x \operatorname{Li}_{2}(x) / x, \zeta(3)$ is the Riemann zeta-function, and $\left[\ln ^{n}(1-x) /(1-x)\right]_{+}$, with $n=0, \ldots, 3$, are plus-distributions defined in the standard way.

We also worked out the normalized photon energy spectrum $1 / G_{77}(0, \mu) \cdot d G_{77}(z, \mu) / d z$, and after setting $\mu=m_{b}$ in our result, we find complete agreement with a recent paper by Melnikov and Mitov [10].

\subsection{Comparison of NLO and NNLO results}

The left frame of Fig. 1 shows the dependence of the spectrum $d G\left(z, m_{b}\right) / d z$ on the rescaled photon energy $z$ at NLO (dashed curve) and NNLO (solid curve). Also plotted is the NLO contribution supplemented by those NNLO terms which are proportional to $\alpha_{s}^{2} \beta_{0}$ (dotted curve). These terms, already worked out in [20], are often called Brodsky-Lepage-Mackenzie (BLM) terms [21]. They arise from the contributions $\sim \alpha_{s}^{2} N_{L}$, after replacing $N_{L} \rightarrow-3 \beta_{0} / 2$ according to the procedure of naive non-abelianization [22]. As seen in the figure, the BLM terms provide the dominant part of the $O\left(\alpha_{s}^{2}\right)$ corrections. As pointed out in [8], one should stress that this statement refers to the scheme where the decay width is written as in (2.1), i.e. with the combination $\bar{m}_{b}^{2}(\mu) m_{b}^{3}$ explicitly appearing.

The behavior of the non-BLM corrections was discussed in detail in [10] and since we agree with their findings, we do not repeat this discussion. However, when comparing the left frame of our Fig. 1 with Fig. 1 of [10], the reader should bear in mind that the spectrum shown in the latter is normalized to the fully integrated quantity $G_{77}\left(0, m_{b}\right)$, followed by a 

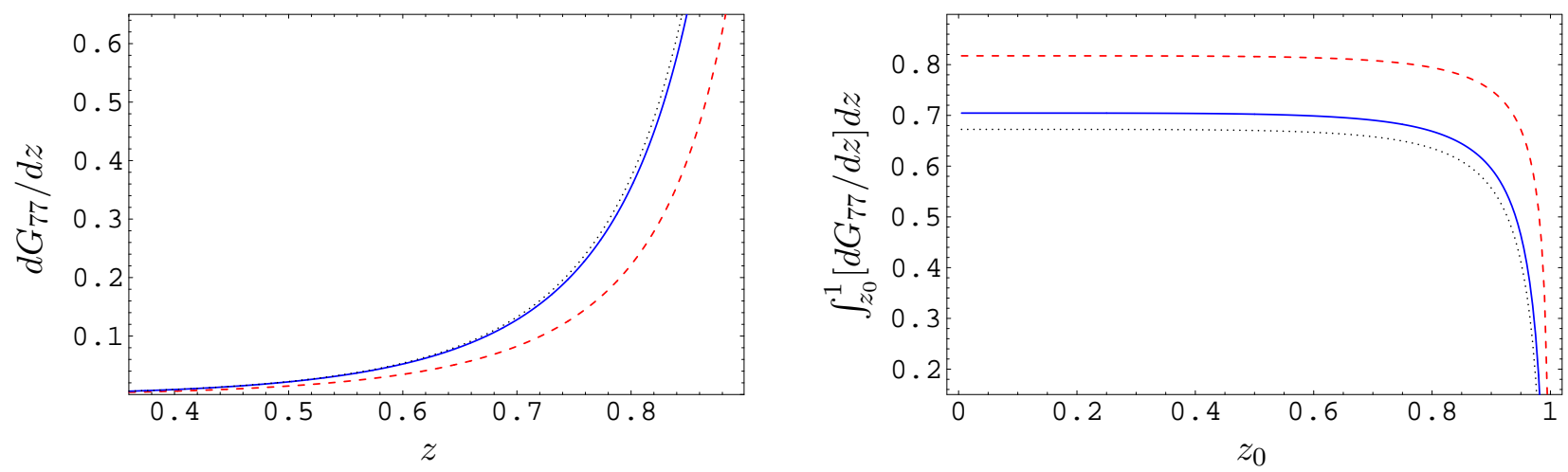

Figure 1: The spectrum $d G_{77}\left(z, m_{b}\right) / d z$ as a function of $z$ (left) and $\int_{z_{0}}^{1}\left[d G_{77} / d z\right] d z$ as a function of $z_{0}$ (right) at NLO (red dashed curve) and NNLO (blue solid curve) for $N_{L}=4$, $N_{H}=1$ and $\alpha_{s}\left(m_{b}\right)=0.22$. The black dotted curve is the BLM approximation of the NNLO result.

consistent expansion in powers of $\alpha_{s}$. As a result the NLO and BLM curves are equal in both figures, but the NNLO approximation lies below the BLM curve in the left frame of our Fig. 1, while it lies above the BLM approximation in the case of the normalized spectrum shown in Fig. 1 of [10].

In the right frame of Fig. 1 we display the quantity $\int_{z_{0}}^{1}\left[d G_{77} / d z\right] d z$ as a function of the (rescaled) photon energy cut-off $z_{0}$. As can be seen, the difference between the NNLO and the BLM result is larger than in the left plot. This is due to the fact that the endpoint contributions at $z=1$ enter this integrated quantity, for which the difference between the BLM approximation and the full NNLO contributions is somewhat larger. But still the BLM terms provide a good approximation also in this case.

\subsection{Change of bottom mass scheme}

The above results have been obtained for a pole $b$ quark mass, that is known to be affected by a leading infrared renormalon and generally leads to slowly converging perturbative expansions. In order to consider a change of scheme for this parameter, it is convenient to use the normalized photon energy spectrum, namely

$$
f(z, \mu) \equiv \frac{\frac{d G_{77}(z, \mu)}{d z}}{G_{77}(0, \mu)}=\delta(1-z)+\frac{\alpha_{s}(\mu)}{\pi} C_{F} f^{(1)}(z)+\left(\frac{\alpha_{s}(\mu)}{\pi}\right)^{2} C_{F} f^{(2)}(z, \mu)+O\left(\alpha_{s}^{3}\right)
$$

A redefinition of the $b$ quark mass $m_{b} \rightarrow m_{b}^{X}-\delta m_{b}^{X}$, where $m_{b}^{X}$ is the $b$ mass in the scheme $X$, leads to a rescaling of the variable $z \rightarrow z^{X}\left(1+\delta m_{b}^{X} / m_{b}^{X}\right)$. A meaningful comparison of different mass schemes can be made at the level of moments of the spectrum or fraction of events. They are physical observables and are expected to have small and computable non-perturbative corrections. Several calculations of these quantities exist [14,23, 24], but they do not always include all the information coming from the $O\left(\alpha_{s}^{2}\right)$ calculation of the spectrum. In the following we drop the $\mu$ dependence from all formulas, set $\mu=m_{b}$, and use $\alpha_{s}\left(m_{b}\right)=0.22$ in the numerics. 
Let us consider for instance the fraction $R\left(E_{0}\right)$ of events with photon energy above a cut $E_{0}$. Neglecting as usual contributions from operators other than $O_{7}$, the perturbative expansion for this quantity,

$$
R\left(E_{0}\right)=1-\int_{0}^{z_{0}} d z f(z), \quad \text { where } \quad z_{0}=\frac{2 E_{0}}{m_{b}},
$$

depends on the mass scheme adopted. In particular, the integral above receives contributions that start at $O\left(\alpha_{s}\right)$. In the pole mass scheme with $m_{b}=4.8 \mathrm{GeV}$ and $E_{0}=1.8 \mathrm{GeV}$ one obtains

$$
\left.R(1.8 \mathrm{GeV})\right|_{\text {pole }}=1-0.0145-0.0085-0.0019+\cdots=0.9751+\cdots
$$

where we have listed separately the tree-level component, the NLO term, the BLM result, and the non-BLM terms at NNLO. As typical in $b$ decays, the large value of the BLM term suggests an optimal scale for $\alpha_{s}$ of $\mathrm{O}(1-2 \mathrm{GeV})$. The inherently large uncertainty in the determination of the pole mass implies a significant uncertainty in $R\left(E_{0}\right)$. For instance, using $m_{b}=5.0 \mathrm{GeV}$ we obtain $\left.R(1.8 \mathrm{GeV})\right|_{\text {pole }}=0.9811$.

Let us repeat the same calculation in a different scheme $X$ for the $b$ mass. Apart from the change in the numerical value of the mass in the evaluation of $z_{0}$, there is an extra NNLO (non-BLM) term

$$
\delta R^{X}\left(E_{0}\right)=-\frac{\alpha_{s}}{\pi} C_{F} z_{0}^{X} \frac{\delta m_{b}^{X}}{m_{b}^{X}} f^{(1)}\left(z_{0}^{X}\right) .
$$

To illustrate the numerical difference due to a change of scheme in $R\left(E_{0}\right)$, we consider here the kinetic mass of the bottom quark [25], also employed in [14]. At $O\left(\alpha_{s}^{2}\right)$ the kinetic mass is related to the pole mass by [26]

$$
\begin{aligned}
m_{b}^{\mathrm{kin}}\left(\mu_{\mathrm{kin}}\right)-m_{b}=- & \frac{\alpha_{s}\left(m_{b}\right)}{\pi} C_{F}\left(\frac{4}{3} \mu_{\text {kin }}+\frac{\mu_{\text {kin }}^{2}}{2 m_{b}^{\text {kin }}\left(\mu_{\text {kin }}\right)}\right) \\
-\left(\frac{\alpha_{s}}{\pi}\right)^{2} C_{F}[ & \left(\beta_{0}\left(\frac{4}{3}-\frac{1}{2} \ln \frac{2 \mu_{\text {kin }}}{m_{b}}\right)-C_{A}\left(\frac{\pi^{2}}{6}-\frac{13}{12}\right)\right) \frac{4}{3} \mu_{\text {kin }} \\
& \left.+\left(\beta_{0}\left(\frac{13}{12}-\frac{1}{2} \ln \frac{2 \mu_{\text {kin }}}{m_{b}}\right)-C_{A}\left(\frac{\pi^{2}}{6}-\frac{13}{12}\right)\right) \frac{\mu_{\text {kin }}^{2}}{2 m_{b}}\right] .
\end{aligned}
$$

Here $\mu_{\text {kin }}$ is a Wilsonian cutoff that factorizes soft and hard gluons; the standard choice is $\mu_{\text {kin }}=1 \mathrm{GeV}$. Inserting (2.21) in $(2.20)$ and employing $m_{b}^{\text {kin }}(1 \mathrm{GeV})=4.6 \mathrm{GeV}$, as suggested by recent fits to the semileptonic moments [13], we obtain

$$
\left.R(1.8 \mathrm{GeV})\right|_{\text {kin }}=1-0.0196-0.0118-0.0025+0.0042+\cdots=0.9703+\cdots
$$

where the last contribution has a positive sign and comes from (2.20). The result in the kinetic scheme is compatible with the one in the pole scheme (2.19), that is expected however to have larger higher order corrections. Eq. (2.22) can be compared with the simple estimate given in [10]: the non-BLM correction has the same magnitude as quoted in [10], but differs for the sign once the additional shift (2.20) is taken into account. As suggested in [10], one 
can write the perturbative expansion in terms of $\alpha_{s}$ at a scale lower than $m_{b}$, improving the apparent convergence of the series, and increasing (by up to a factor $\sim 2.5$ ) the relative importance of the non-BLM term. A consistent implementation of the kinetic scheme has been presented, up to $O\left(\alpha_{s}^{2} \beta_{0}\right)$, in [14]: besides the use of the kinetic bottom mass, it involves a proper definition of the non-perturbative matrix elements of higher dimensional operators and a cut on soft-gluon radiation that modifies the shape of the spectrum close to the endpoint. A complete implementation of these effects is beyond the scope of the present paper, but we expect (2.22) to show the likely impact of the $O\left(\alpha_{s}^{2}\right)$ photon spectrum on the kinetic scheme calculation of $R\left(E_{0}\right)$.

Finally, we consider the truncated first moment of the normalized spectrum in the pole and kinetic mass schemes, neglecting all non-perturbative corrections and the contributions from operators other than $O_{7}$. It can be written in the on-shell scheme as

$$
\left\langle E_{\gamma}\right\rangle_{E_{\gamma}>E_{0}}=\frac{m_{b}}{2}\left[1-\int_{z_{0}}^{1} d z(1-z) f(z)\left(1+\int_{0}^{z_{0}} d y f(y)\right)+O\left(\alpha_{s}^{3}\right)\right] .
$$

Using the same numerical inputs as above, the pole scheme expansion is

$$
\left\langle E_{\gamma}\right\rangle_{E_{\gamma}>1.8 \mathrm{GeV}} \simeq \frac{m_{b}}{2}[1-0.0246-0.0242+0.0037-0.0004]=2.291 \mathrm{GeV}
$$

where we have listed separately the tree-level, NLO, BLM, NNLO non-BLM, and the contribution of the interference (last) term in (2.23). The relative weight of the BLM contribution is even larger than in the previous example, as expected since the first moment probes a region of smaller gluon energies.

A change of bottom mass scheme in the first moment leads to the extra term

$$
\delta\left\langle E_{\gamma}\right\rangle_{E_{\gamma}>E_{0}}^{X}=-\frac{\delta m_{b}^{X}}{2}\left[1-C_{F} \frac{\alpha_{s}}{\pi}\left(z_{0}^{X}\left(1-z_{0}^{X}\right) f^{(1)}\left(z_{0}^{X}\right)+\int_{z_{0}^{X}}^{1} d z(1-z) f^{(1)}(z)\right)\right],
$$

where $\delta m_{b}^{X}$ now includes the $O\left(\alpha_{s}^{2}\right)$ term, and the r.h.s. must be expanded up to $O\left(\alpha_{s}^{2}\right)$. The scheme change affects already the NLO calculation and at NNLO contributes also to the BLM term. The numerical result in the kinetic scheme is

$$
\begin{array}{r}
\left\langle E_{\gamma}\right\rangle_{E_{\gamma}>1.8 \mathrm{GeV}} \simeq \frac{m_{b}^{\mathrm{kin}}(1 \mathrm{GeV})}{2}[1-0.0234-0.0234+0.0038-0.0005 \\
\quad+0.0293+0.0296-0.0035-0.0016]=2.324 \mathrm{GeV}
\end{array}
$$

where the last four entries are the NLO, BLM, NNLO non-BLM mass shifts, and the mixed term in (2.25). Once again, the result is consistent with the one in the on-shell scheme, that is however subject to a larger uncertainty; it is also consistent with the experimental value by the Belle collaboration [27]: $\left\langle E_{\gamma}\right\rangle_{E_{\gamma}>1.8 \mathrm{GeV}}=2.292 \pm 0.026 \pm 0.034 \mathrm{GeV}$. We stress that our calculation in the kinetic scheme is not complete and that non-perturbative effects have not been included (see [14]). We agree with [10] for the part included in that paper, which does not properly consider the change of scheme. Our result for the non-BLM NNLO contribution to $(2.26)$ is $m_{b}^{\mathrm{kin}}(1 \mathrm{GeV}) / 2(0.0038-0.0005-0.0035-0.0016) \simeq-4 \mathrm{MeV}$. Even if we rescale it to account for a lower $\alpha_{s}$ scale, this has the opposite sign and is much smaller than the estimate in [10]. It can be expected to shift the $b$ quark mass extracted from the first moment by less than $15 \mathrm{MeV}$, well beyond present sensitivity. On the other hand, it is clear that a complete NNLO analysis of the $\bar{B} \rightarrow X_{s} \gamma$ moments based on the spectrum presented here will have a smaller perturbative uncertainty. 

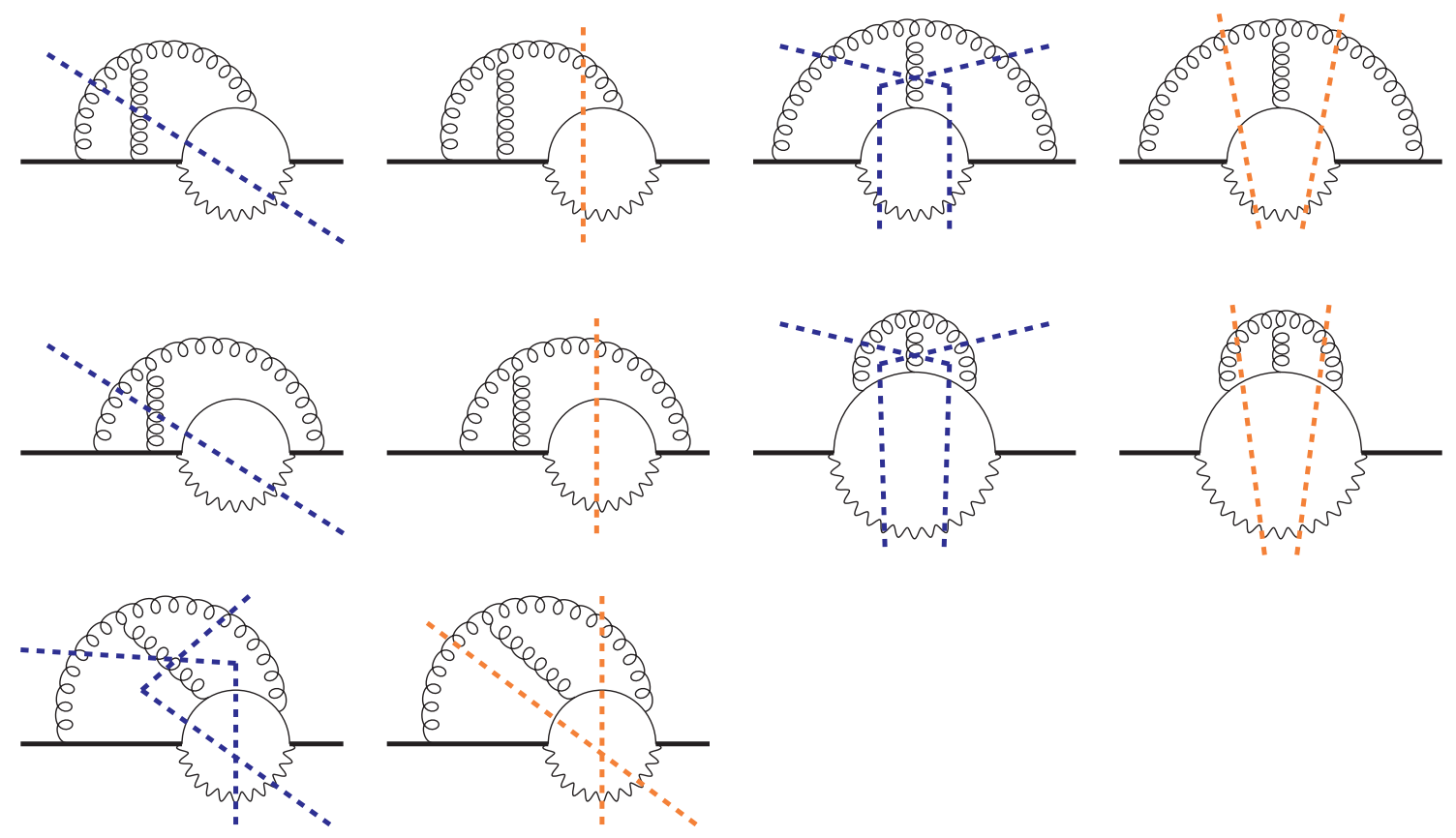

Figure 2: Diagrams contributing to $\Gamma_{1 \rightarrow n}$ for $n=3$ and 4 at $O\left(\alpha_{s}^{2}\right)$. We draw here only the nonabelian diagrams. Thick lines represent the massive $b$ quark, thin lines the massless $s$ quark, wavy lines photons and curly lines gluons. Dashed lines separate the original Feynman diagrams which enter the squared amplitude $\left|M_{n}\right|^{2}$. The dashed blue lines indicate cuts contributing to the $b \rightarrow s \gamma g g$ process, and the dashed orange lines indicate cuts contributing to the $b \rightarrow s \gamma g$ process. Possible left-right reflected diagrams are not shown. See text for more details.

\section{Details of the calculation}

Our goal is to calculate the NNLO corrections to the quantity $d G_{77}(z, \mu) / d z$ introduced in (2.4), and, as stated in the previous section, to do so we only have to calculate contributions with three and four particles in the final state. Hence, we restrict the following discussion to the cases $n=3$ and 4 [see (2.5)].

We start by considering the general expression for the decay rate of a massive $b$ quark with momentum $p_{b}$ into $n=3,4$ massless final-state particles with momenta $k_{i}$,

$$
\begin{aligned}
\Gamma_{1 \rightarrow n}= & \frac{1}{2 m_{b}}\left(\prod_{i=1}^{n} \int \frac{d^{d-1} k_{i}}{(2 \pi)^{d-1} 2 E_{i}}\right)(2 \pi)^{d} \delta^{(d)}\left(p_{b}-\sum_{i=1}^{n} k_{i}\right)\left|M_{n}\right|^{2} \\
= & \frac{1}{2 m_{b}}(2 \pi)^{n}\left(\prod_{i=1}^{n-1} \int \frac{d^{d} k_{i}}{(2 \pi)^{d}} \delta\left(k_{i}^{2}\right) \theta\left(k_{i}^{0}\right)\right) \\
& \times \delta\left(\left(p_{b}-\sum_{i=1}^{n-1} k_{i}\right)^{2}\right) \theta\left(p_{b}^{0}-\sum_{i=1}^{n-1} k_{i}^{0}\right)\left|M_{n}\right|^{2},
\end{aligned}
$$

where the squared Feynman amplitude $\left|M_{n}\right|^{2}$ is always understood as summed over final spin-, polarization- and color states, and averaged over the spin directions and colors of the decaying $b$ quark. For $n=4$ it also includes a possible factor of $1 / 2$ if there are two identical particles in the final state. Furthermore, $d=4-2 \varepsilon$ denotes the space-time dimension that 


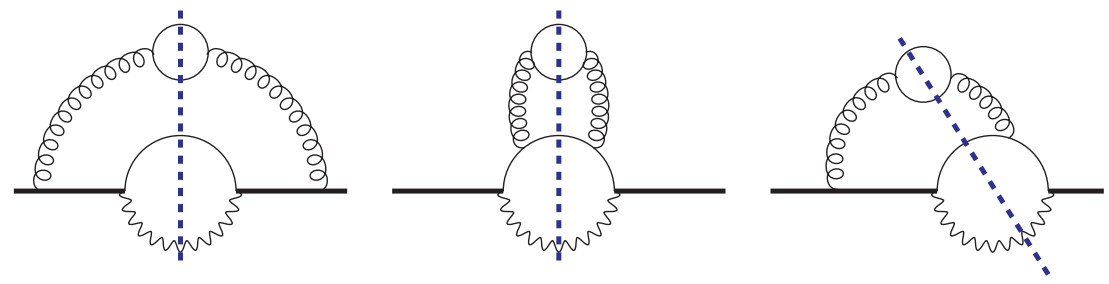

Figure 3: Same as in Fig. 2 for diagrams with a closed light quark loop. Similar diagrams with a closed gluon loop are also present. The dashed blue lines indicate cuts contributing to the $b \rightarrow s \gamma q \bar{q}(q \in\{u, d, c, s\})$ and $b \rightarrow s \gamma g g$ processes. Concerning diagrams with closed ghost loops, see text. Note that three-particles cuts of the diagrams above are zero in dimensional regularization.

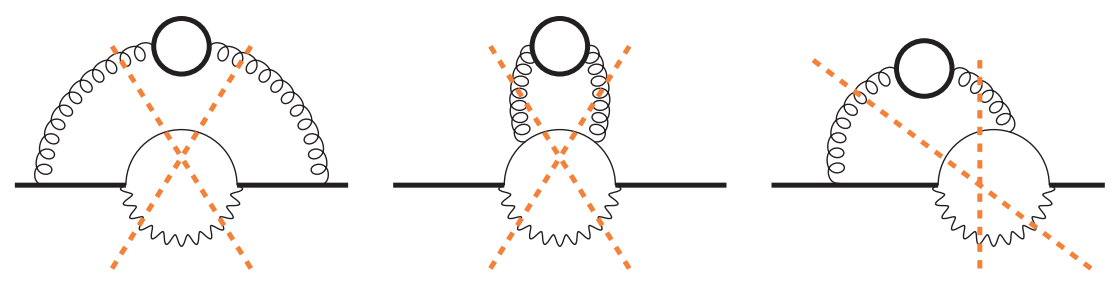

Figure 4: Same as in Fig. 2 for diagrams including a closed bottom quark loop. The dashed orange lines indicate cuts contributing to the $b \rightarrow s \gamma g$ process. Four-particles cuts of these diagrams are kinematically not allowed.

we use to regulate the ultraviolet, infrared and collinear singularities. We also stress that we used the Feynman gauge for the gluon propagator.

Since we are interested in the photon energy spectrum, we fix the photon energy by inserting in the phase-space integrand on the r.h.s. of (3.1) a factor

$$
\delta\left(E_{\gamma}-\frac{p_{b} \cdot p_{\gamma}}{m_{b}}\right)=2 m_{b} \delta\left(\left(p_{b}+p_{\gamma}\right)^{2}-(1+z) m_{b}^{2}\right)
$$

Then, after performing the phase-space integrations over the full range, $\Gamma_{1 \rightarrow n}$ coincides with $d G_{77}^{1 \rightarrow n}(z, \mu) / d z$ given in (2.4) up to a constant factor.

The optical theorem relates the $b$ quark decay rate we are interested in to the imaginary parts of three-loop $b$ quark self-energy diagrams. The contribution of a specific physical cut of the three-loop diagrams to the imaginary part of the $b$ quark self-energy can be evaluated using the Cutkosky rules [28], resulting in precisely the same integrals that appear on the r.h.s. of (3.1). The various contributions to $d G_{77}^{1 \rightarrow n}(z, \mu) / d z$ can therefore be easily represented in terms of cut three-loop diagrams. Figs. 2-5 show the physical cuts that give non-vanishing contributions to $d G_{77}^{1 \rightarrow n}(z, \mu) / d z$.

The individual contributions to the decay rate (or, equivalently, each cut-diagram) can 

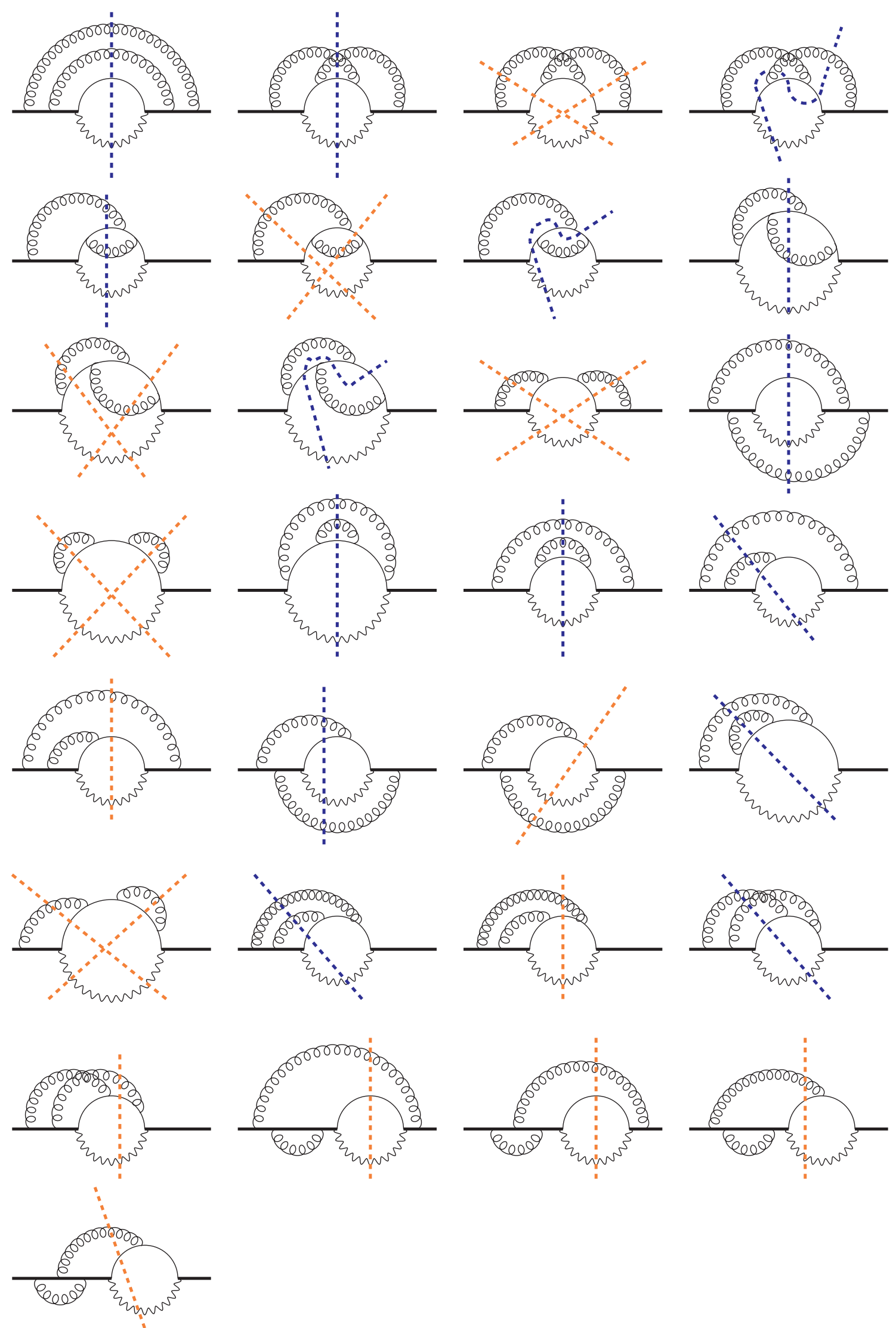

Figure 5: Same as in Fig. 2 for abelian diagrams without a closed quark loop. The color code is the same as in the figures 2,3 and 4 . 
now be evaluated with the technical tools usually employed in the calculation of multiloop Feynman diagrams. To this end, we rewrite all delta functions appearing in the phase-space representation (3.1) as well as the one from the kinematic constraint in (3.2) as a difference of propagators $[29,30]$,

$$
\delta\left(q^{2}-m^{2}\right)=\frac{1}{2 \pi i}\left(\frac{1}{q^{2}-m^{2}-i 0}-\frac{1}{q^{2}-m^{2}+i 0}\right) .
$$

The expressions for $\Gamma_{1 \rightarrow n}$ can then be reduced algebraically by means of the systematic Laporta algorithm [31], based on the integration-by-part identities (IBPs) first proposed in $[32,33]$. Basically, the Laporta algorithm allows one to express the various contributions to the integral in (3.1) (i.e. the various cut diagrams in Figs. 2-5) as linear combinations of a small number of simpler integrals, usually referred to as the master integrals (MIs) of the problem. The coefficients in front of the MIs are ratios of polynomials of the kinematic variables present in the problem; in the case under study, the only kinematic variable is $z$. The reduction procedure is simplified by the fact that all the integrals in which one of the cut propagators disappears from the integrand, or is raised to a negative power, are zero, because in this cases the $\pm i 0$ prescription becomes irrelevant. A further comment concerning the application of the IBPs to the calculation of phase-space integrals is in order. It is not often stressed that the propagators originating from phase-space integrations via (3.3) are always accompanied by a factor $\theta\left(q_{0}\right)$, where $q_{0}$ is the energy flowing in the propagator. In building the IBPs it is necessary to take the derivative of the integrand of a given diagram with respect to the integration momenta. When the derivative $\partial / \partial q_{0}$ acts on $\theta\left(q_{0}\right)$, the latter transforms into a delta function and hence terms like

$$
\delta\left(q_{0}\right)\left(\frac{1}{q^{2}-m^{2}-i 0}-\frac{1}{q^{2}-m^{2}+i 0}\right)=\delta\left(q_{0}\right)\left(\frac{-1}{\vec{q}^{2}+m^{2}+i 0}-\frac{-1}{\vec{q}^{2}+m^{2}-i 0}\right)
$$

are produced. Obviously, the $\pm i 0$ prescription is of no relevance here and the two terms in the parenthesis cancel. Thus terms where the derivative acts on theta functions do not contribute to the IBPs. Delta functions enforcing kinematic conditions, like the one in (3.2), are not multiplied by theta functions. We made use of two automatic implementations of the Laporta algorithm: AIR [34] based on Maple [35] and an independent Mathematica [36] code.

Once the reduction to MIs has been completed, the propagators introduced via (3.3) are reconverted into delta functions and the MIs are evaluated. After introducing appropriate parameterizations for the three- and the four-particle phase-space [37,38] (and Feynman parameters in order to perform the loop integration if the MI includes a closed loop without cut propagators), the simpler integrals can be calculated analytically after expanding in $\varepsilon$. To obtain analytic expressions for the more difficult ones, it is convenient to employ the differential equation method [39] as described in detail in [29,30]. As an independent check we also computed all the MIs numerically by means of the sector decomposition method [40] (see [9] for further details).

The color factors for the individual diagrams shown in Figs. 2-5 are listed in Table I of [8]. The contribution of the diagrams which are not left-right symmetric must be multiplied by a factor 2. Notice that we also included diagrams which arise by replacing the quark loop in Fig. 3 by a gluon loop. The four-particle cuts of these diagrams can be calculated in two ways: (a) using the complete expression for the polarization sum of the two transverse gluons, or, 
(b) replacing the polarization sums of the gluons by the negative of the metric tensor; in this case, the emission of ghost particles (corresponding to four-particle cuts through diagrams with a closed ghost loop) has to be taken into account. We convinced ourselves in [9] that the two procedures lead to the same result. In the present paper we used procedure (b). As pointed out in [8], the contributions of the diagrams with a closed gluon/ghost loop can be obtained from those involving a closed light quark loop by replacing (in the Feynman gauge)

$$
T_{R} N_{L} \rightarrow-C_{A}\left(\frac{5}{4}+\frac{\varepsilon}{2}+O\left(\varepsilon^{2}\right)\right) .
$$

The diagrams in Figs. 2-5 involve IR, collinear and UV singularities, that appear in the intermediate steps of the calculation as poles in the dimensional regulator $\varepsilon$. Some of the cuts generate not only single, but also double poles; the latter, however, cancel in the sum of all the cuts belonging to a given $b$ quark self-energy diagram. The sum of all diagrams has a single pole that gets removed when adding the counterterm contributions.

We conclude this section by summarizing the renormalization procedure adopted; it coincides with the one employed in $[8,9]$. As explained after (2.6), our calculation can be restricted to $z \neq 1$. In this case all counterterms of $O\left(\alpha_{s}^{2}\right)$ (i.e. those contributing to $H^{(2)}$ defined in (2.10)) are induced by renormalizing the various parameters and wave functions in the process $b \rightarrow s \gamma g$. To get the counterterms proportional to the color factors $C_{A}$ and $T_{R} N_{L}$, only the renormalization of the strong coupling constant is needed. For the term proportional to $T_{R} N_{H}$ we need the renormalization of the strong coupling constant and that of the gluon wave function. In order to renormalize the contribution proportional to $C_{F}$, one needs also to consider the renormalization of the operator $O_{7}$, the renormalization of the bottom and strange quark wave functions, and of the bottom quark mass. The renormalization constants, which are only needed to $O\left(\alpha_{s}\right)$ precision in our calculation, are fixed as follows:

i) the strong coupling constant is renormalized using the $\overline{\mathrm{MS}}$ scheme:

$$
Z_{\alpha}^{\overline{\mathrm{MS}}}=1+\left(T_{R} N_{F} K_{\alpha}^{(1)}+C_{A} K_{\alpha}^{(2)}\right) \frac{\alpha_{s}(\mu)}{\pi}+O\left(\alpha_{s}^{2}\right), \quad K_{\alpha}^{(1)}=\frac{1}{3 \varepsilon}, \quad K_{\alpha}^{(2)}=-\frac{11}{12 \varepsilon}
$$

ii) the $b$ quark mass contained in the operator $O_{7}$ and the Wilson coefficient $C_{7}^{\text {eff }}$ are renormalized in the $\overline{\mathrm{MS}}$ scheme in our calculation. In the present application we only need the product of these two renormalization constants which is given by

$$
Z_{m_{b}}^{\overline{\mathrm{MS}}} Z_{77}^{\overline{\mathrm{MS}}}=1+C_{F} K_{77} \frac{\alpha_{s}(\mu)}{\pi}+O\left(\alpha_{s}^{2}\right), \quad K_{77}=\frac{1}{4 \varepsilon}
$$

iii) all the remaining external fields and the $b$ mass are renormalized in the on-shell scheme. The on-shell renormalization constants for the $b$ quark mass and wave function are given by

$$
\begin{array}{ll}
Z_{m_{b}}^{\mathrm{OS}}=1+C_{F} K_{m_{b}} \frac{\alpha_{s}(\mu)}{\pi}+O\left(\alpha_{s}^{2}\right), & K_{m_{b}}=-\frac{3}{4 \varepsilon}-1-\frac{3}{2} L_{\mu}, \\
Z_{2 b}^{\mathrm{OS}}=1+C_{F} K_{2 b} \frac{\alpha_{s}(\mu)}{\pi}+O\left(\alpha_{s}^{2}\right), \quad K_{2 b}=K_{m_{b}} .
\end{array}
$$


For the wave-function renormalization factor of the gluon-field we find

$$
Z_{3}^{\mathrm{OS}}=1+T_{R} N_{H} K_{3} \frac{\alpha_{s}(\mu)}{\pi}+O\left(\alpha_{s}^{2}\right), \quad K_{3}=-\frac{1}{3 \varepsilon}-\frac{2}{3} L_{\mu}
$$

The $s$-quark field renormalization constant is equal to one. [Notice that in our approach the effects of the diagrams in Fig. 4 are taken into account through the renormalization of the gluon wave function.]

Consequently, we find $(z \neq 1)$

$$
\begin{aligned}
H^{(2, \mathrm{a})} & =H^{(2, \mathrm{a}, \mathrm{bare})}+\left(2 K_{77}+K_{2 b}\right) H^{(1, \varepsilon)}-K_{m_{b}} H^{(1, m, \varepsilon)}, \\
H^{(2, \mathrm{na})} & =H^{(2, \text { na, bare })}+K_{\alpha}^{(2)} H^{(1, \varepsilon)}(z) \\
H^{(2, \mathrm{NL})} & =H^{(2, \mathrm{NL}, \text { bare })}+K_{\alpha}^{(1)} H^{(1, \varepsilon)}(z) \\
H^{(2, \mathrm{NH})} & =\left(K_{\alpha}^{(1)}+K_{3}\right) H^{(1, \varepsilon)}
\end{aligned}
$$

where the quantities $H^{(2, i, \text { bare })}(i=\mathrm{a}$, na, NL) are the unrenormalized contributions to the photon energy spectrum corresponding to the diagrams given in Figs. 2, 3 and 5 . The function $H^{(2, \mathrm{NH} \text {,bare })}$ vanishes in our approach as just explained. The functions $H^{(1, \varepsilon)}$ and $H^{(1, m, \varepsilon)}$ include terms of order $\varepsilon$ and their explicit expressions are collected in the Appendix.

\section{Summary}

A precise calculation of the photon energy spectrum in the inclusive $\bar{B} \rightarrow X_{s} \gamma$ decay is crucial for comparing theoretical predictions with measurements. In this paper we calculated the NNLO QCD corrections to the photon energy spectrum induced by the magnetic penguin operator $\mathrm{O}_{7}$. This subset of contributions is likely to provide the leading correction to the photon energy spectrum at $O\left(\alpha_{s}^{2}\right)$. The present calculation represents an independent check of the results of [10], with which we find complete agreement.

The numerical relevance of the results and some application have already been studied in [10] for the normalized photon energy spectrum: in particular, the effect of the non-BLM corrections is minor with respect to the dominant BLM component [19]. This statement also holds for the unnormalized photon energy spectrum $d G_{77} / d z$ shown in the left frame of Fig. 1, as well as for the integrated quantity $\int_{z_{0}}^{1}\left[d G_{77} / d z\right] d z$ shown in the right frame of this figure, provided the decay width is written to be proportional to $\bar{m}_{b}(\mu)^{2} m_{b}^{3}$ as in (2.1).

Furthermore, we investigated the impact of the change of the bottom quark mass from the pole to the kinetic scheme on observables like the first moment of the spectrum $\left\langle E_{\gamma}\right\rangle_{E \gamma>E_{0}}$ and the fraction of events with photon energy above $E_{0}, R\left(E_{0}\right)$. Apart from the change of the numerical values of the $b$ quark mass, these observables receive the additional shifts presented in (2.20) and (2.25). We find that the results in the two schemes are compatible, although the pole scheme is expected to have larger higher order corrections.

In the calculation we employed a number of techniques usually applied to the calculation of multiloop Feynman diagrams, such as the Laporta algorithm, the differential equation method for the analytic evaluation of master integrals, and sector decomposition as an 
independent numerical check of the analytically evaluated master integrals. Several technical aspects of the calculation were discussed in detail in Sec. 3. The same techniques can now be applied to the calculation of the subleading $O\left(\alpha_{s}^{2}\right)$ contributions $\left(\mathrm{O}_{7}, \mathrm{O}_{8}\right)$ and $\left(\mathrm{O}_{8}, \mathrm{O}_{8}\right)$ to the photon energy spectrum of the inclusive radiative $B$ decay.

\section{Acknowledgements}

H. M. A. is partially supported by the ANSEF N 05-PS-hepth-0825-338 program, C. G. and T. E. by the Swiss National Foundation as well as RTN, BBW-Contract No.01.0357 and EC-Contract HPRN-CT-2002-00311 (EURIDICE). P. G. is supported by MIUR under contract 2004021808-009. A. F. is grateful to E. Remiddi for a useful discussion on the use of IBPs in problems involving cut propagators, and to J. Vermaseren for his kind assistance in the use of the algebraic manipulation program FORM [41].

\section{A Functions $\boldsymbol{H}^{(1, \varepsilon)}$ and $\boldsymbol{H}^{(1, m, \varepsilon)}$}

Here we collect the functions $H^{(1, \varepsilon)}$ and $H^{(1, m, \varepsilon)}$ including $O(\varepsilon)$ terms as required by renormalization. For $z \neq 1$, that is with terms proportional to $\delta(1-z)$ dropped, they are given by

$$
\begin{aligned}
H^{(1, \varepsilon)}(z)= & {\left[-\frac{\ln (1-z)}{1-z}-\frac{7}{4} \frac{1}{1-z}-\frac{1+z}{2} \ln (1-z)+\frac{7+z-2 z^{2}}{4}\right]\left(1+4 \varepsilon L_{\mu}\right) } \\
& +\varepsilon\left[\frac{1}{1-z}\left(\frac{\pi^{2}}{3}-\frac{7}{2}-2 \operatorname{Li}_{2}(1-z)\right)+\frac{7}{4} \frac{\ln (1-z)}{1-z}+\frac{3}{2} \frac{\ln ^{2}(1-z)}{1-z}\right. \\
& +\frac{7}{2} \frac{\ln z}{1-z}+\frac{3}{4}(1+z) \ln ^{2}(1-z)-\frac{7+z-2 z^{2}}{4}(\ln (1-z)+2 \ln z) \\
& \left.+(1+z)\left(\frac{\pi^{2}}{6}-\operatorname{Li}_{2}(1-z)\right)+\frac{7+z-2 z^{2}}{2}\right]+O\left(\varepsilon^{2}\right), \\
H^{(1, m, \varepsilon)}(z)= & {\left[3 \frac{\ln (1-z)}{1-z}+\frac{6}{1-z}+(1+z) \ln (1-z)-(6+2 z)\right]\left(1+4 \varepsilon L_{\mu}\right) } \\
& +\varepsilon\left[-\frac{2}{(1-z)^{2}}-\frac{9}{2} \frac{\ln ^{2}(1-z)}{1-z}-12 \frac{\ln (1-z)}{1-z}-12 \frac{\ln z}{1-z}\right. \\
& -2\left[\frac{3}{1-z}+(1+z)\right]\left(\frac{\pi^{2}}{6}-\operatorname{Li}_{2}(1-z)\right)+\frac{8}{1-z}-\frac{3}{2}(1+z) \ln ^{2}(1-z) \\
& +(6+z) \ln (1-z)+(12+4 z) \ln z-6-2 z]+O\left(\varepsilon^{2}\right) .
\end{aligned}
$$




\section{References}

[1] Heavy Flavour Averaging Group, see http://www.slac.stanford.edu/xorg/hfag/.

[2] P. Gambino and M. Misiak, Nucl. Phys. B 611 (2001) 338 [arXiv:hep-ph/0104034].

[3] A. J. Buras and M. Misiak, Acta Phys. Polon. B 33 (2002) 2597 [arXiv:hep-ph/0207131].

[4] M. Misiak and M. Steinhauser, Nucl. Phys. B 683 (2004) 277 [hep-ph/0401041].

[5] M. Gorbahn and U. Haisch, Nucl. Phys. B 713 (2005) 291 [hep-ph/0411071].

[6] M. Gorbahn, U. Haisch and M. Misiak, Phys. Rev. Lett. 95 (2005) 102004 [hep$\mathrm{ph} / 0504194]$.

[7] K. Bieri, C. Greub and M. Steinhauser, Phys. Rev. D 67 (2003) 114019 [arXiv:hep$\mathrm{ph} / 0302051]$.

[8] I. Blokland, A. Czarnecki, M. Misiak, M. Slusarczyk and F. Tkachov, Phys. Rev. D 72 (2005) 033014 [arXiv:hep-ph/0506055].

[9] H. M. Asatrian, A. Hovhannisyan, V. Poghosyan, T. Ewerth, C. Greub and T. Hurth, Nucl. Phys. B 749 (2006) 325 [arXiv:hep-ph/0605009].

[10] K. Melnikov and A. Mitov, Phys. Lett. B 620 (2005) 69 [arXiv:hep-ph/0505097].

[11] M. Czakon, U. Haisch, M. Misiak, in progress; U. Haisch, talk at the workshop Flavour in the LHC era, see http://flavlhc. web.cern.ch/flavlhc/.

[12] H. M. Asatrian, C. Greub, A. Hovhannisyan, Y. P. Inst., T. Hurth and V. Poghosyan, Phys. Lett. B 619 (2005) 322 [hep-ph/0505068].

[13] O. Buchmuller and H. Flacher, Phys. Rev. D 73 (2006) 073008 [hep-ph/0507253]; C. W. Bauer, Z. Ligeti, M. Luke, A. V. Manohar and M. Trott, Phys. Rev. D 70 (2004) 094017 [hep-ph/0408002]; M. Neubert, Phys. Rev. D 72 (2005) 074025 [hep$\mathrm{ph} / 0506245]$.

[14] D. Benson, I. I. Bigi and N. Uraltsev, Nucl. Phys. B 710 (2005) 371 [hep-ph/0410080].

[15] I. I. Y. Bigi, M. A. Shifman, N. G. Uraltsev and A. I. Vainshtein, Int. J. Mod. Phys. A 9 (1994) 2467 [hep-ph/9312359]; M. Neubert, Eur. Phys. J. C 40 (2005) 165 [hepph/0408179]; J. R. Andersen and E. Gardi, JHEP 0506 (2005) 030 [hep-ph/0502159].

[16] E. Gardi, JHEP 0502 (2005) 053 [hep-ph/0501257].

[17] T. Becher and M. Neubert, Phys. Lett. B 633 (2006) 739 [hep-ph/0512208] and Phys. Lett. B 637 (2006) 251 [hep-ph/0603140].

[18] A. Ali and C. Greub, Z. Phys. C 49 (1991) 431; A. Ali and C. Greub, Phys. Lett. B 259 (1991) 182; A. Ali and C. Greub, Phys. Lett. B 361 (1995) 146 [arXiv:hep-ph/9506374]; N. Pott, Phys. Rev. D 54 (1996) 938 [arXiv:hep-ph/9512252]. 
[19] Z. Ligeti, M. E. Luke, A. V. Manohar and M. B. Wise, Phys. Rev. D 60 (1999) 034019 [arXiv:hep-ph/9903305].

[20] Z. Ligeti, M. E. Luke, A. V. Manohar and M. B. Wise, Phys. Rev. D 60 (1999) 034019 [arXiv:hep-ph/9903305].

[21] S. J. Brodsky, G. P. Lepage and P. B. Mackenzie, Phys. Rev. D 28 (1983) 228.

[22] M. Beneke and V. M. Braun, Phys. Lett. B 348 (1995) 513 [ArXiv:hep-ph/9411229].

[23] J. R. Andersen and E. Gardi, JHEP 0506 (2005) 030 [arXiv:hep-ph/0502159].

[24] M. Neubert, Phys. Rev. D 72 (2005) 074025 [arXiv:hep-ph/0506245].

[25] I. I. Y. Bigi, M. A. Shifman, N. Uraltsev and A. I. Vainshtein, Phys. Rev. D 56 (1997) 4017 [arXiv:hep-ph/9704245] and Phys. Rev. D 52 (1995) 196 [arXiv:hep-ph/9405410].

[26] A. Czarnecki, K. Melnikov and N. Uraltsev, Phys. Rev. Lett. 80 (1998) 3189 [arXiv:hep$\mathrm{ph} / 9708372]$.

[27] P. Koppenburg et al. [Belle Coll.], Phys. Rev. Lett. 93 (2004) 061803 [arXiv:hepex/0403004]; see also B. Aubert et al. [Babar Coll.], hep-ex/0607071.

[28] R. E. Cutkosky, J. Math. Phys. 1 (1960) 429; M. J. G. Veltman, Physica 29 (1963) 186; E. Remiddi, Helv. Phys. Acta 54 (1982) 364.

[29] C. Anastasiou and K. Melnikov, Nucl. Phys. B 646 (2002) 220 [arXiv:hep-ph/0207004].

[30] C. Anastasiou, L. J. Dixon, K. Melnikov and F. Petriello, Phys. Rev. D 69 (2004) 094008 [arXiv:hep-ph/0312266].

[31] S. Laporta, Int. J. Mod. Phys. A 15 (2000) 5087 [arXiv:hep-ph/0102033].

[32] F. V. Tkachov, Phys. Lett. B 100 (1981) 65.

[33] K. G. Chetyrkin and F. V. Tkachov, Nucl. Phys. B 192 (1981) 159.

[34] C. Anastasiou and A. Lazopoulos, JHEP 0407 (2004) 046 [arXiv:hep-ph/0404258].

[35] Maple 9.5, Copyright 2004 by Maplesoft.

[36] Mathematica 5.0, Copyright 2003 by Wolfram Research.

[37] A. Gehrmann-De Ridder, T. Gehrmann and G. Heinrich, Nucl. Phys. B682 (2004) 265 [arXiv:hep-ph/0311276].

[38] C. Anastasiou, K. Melnikov and F. Petriello, Phys. Rev. D 69 (2004) 076010 [arXiv:hep$\mathrm{ph} / 0311311]$.

[39] E. Remiddi, Nuovo Cim. A 110 (1997) 1435.

[40] T. Binoth and G. Heinrich, Nucl. Phys. B 680 (2004) 375 [arXiv:hep-ph/0305234].

[41] J. A. M. Vermaseren, arXiv:math-ph/0010025. 